\title{
Analytic sensing: direct recovery of point sources from planar Cauchy boundary measurements
}

\author{
D. Kandaswamy ${ }^{a}$, T. Blu ${ }^{a}$ and D. Van De Ville ${ }^{a}$ \\ ${ }^{a}$ Biomedical Imaging Group, École Polytechnique Fédérale de Lausanne, CH-1015 Lausanne, \\ Switzerland
}

\begin{abstract}
Inverse problems play an important role in engineering. A problem that often occurs in electromagnetics (e.g. EEG) is the estimation of the locations and strengths of point sources from boundary data.

We propose a new technique, for which we coin the term "analytic sensing". First, generalized measures are obtained by applying Green's theorem to selected functions that are analytic in a given domain and at the same time localized to "sense" the sources. Second, we use the finite-rate-of-innovation framework to determine the locations of the sources. Hence, we construct a polynomial whose roots are the sources' locations. Finally, the strengths of the sources are found by solving a linear system of equations. Preliminary results, using synthetic data, demonstrate the feasibility of the proposed method.
\end{abstract}

Keywords: analytic functions, Laplace's equation, annihilating filters, source localization

\section{INTRODUCTION}

A well-known inverse problem is source imaging from boundary Cauchy data, where the underlying model is the Laplace equation. Unfortunately, such a problem is ill-posed and thus, in order to have a unique solution, we need additional restrictions on the source configuration. Typically, one can restrict the class of source distributions by imposing smoothness properties (e.g., Tikhonov regularization ${ }^{2}$ ) or by imposing a parametric source model.

Parametric solutions are very useful for applications that consider point sources. This is often the case in the electromagnetic setting such as for electroencephalography (EEG), for example, the localization of epileptic foci which can be reasonably modeled by point (dipolar) sources. ${ }^{3}$ Most localization techniques depend on the forward model; i.e., computing the boundary data for a given source configuration and iteratively fitting its parameters by least-squares optimization. ${ }^{4}$ However, the non-linear relationship between the sources' positions and the boundary data imply the existence of local minima and make the solution very sensitive to the initial guess. Hence, recovery of the parametric sources is often limited to single-dipole models.

The mathematical uniqueness of the solution has been proven, ${ }^{5}$ and stability results are available for the case of dipolar and point sources in $2 \mathrm{D}^{4,6}$ and $3 \mathrm{D} .^{7}$ In this work, we propose a novel, non-iterative algorithm for a parametric model to recover point sources.

The main idea is to express the inverse problem at hand as a generalized sampling problem. The generalized samples are obtained by applying Green's theorem with multiple, well-chosen test functions that are analytic in the domain. We name this procedure "analytic sensing" as the test functions capture ("sense") the local influence of the sources. In practice these measures can be obtained by computing a surface integral with the boundary data. Next, we generalize the "annihilating filter" approach as proposed in $^{1}$ to reconstruct the locations of the point sources. Once the locations are known, the corresponding strengths are obtained through a linear system of equations.

Further author information:

Kandaswamy D.: E-mail: djano.kandaswamy@epfl.ch, Telephone: +41223725215

Blu T. E-mail: Thierry.Blu@epfl.ch, Telephone: +41 216935172

Van De Ville D., E-mail: Dimitri.VanDeVille@epfl.ch, Telephone: +41223725215 


\section{PROBLEM SETTING}

Let us consider a closed, 2D domain $\Omega$ with boundary $\partial \Omega$. Moreover, consider a source distribution $\rho$ in $\Omega$.The source distribution manifests itself on the boundary $\Omega$, such that:

$$
-\nabla \cdot(\sigma \nabla V)=\rho,
$$

where $\sigma$ expresses the inhomogeneity of the environment $\Omega$ and $V$ is the manifestation of the source configuration $\rho$. In this paper we consider $\sigma$ to be constant, that is $\sigma=1$. Hence, (1) is equivalent to Laplace's equation, which can be written as follows:

$$
-\Delta V=\rho .
$$

There are many physical problems that can be modeled using (1), e.g. the electromagnetic setting. In that case equation (2) relates the generated potential differences, $\phi$, to the electric source distribution, $\rho$, in a conductor with conductivity profile $\sigma$. Moreover, there is a boundary condition which states that no current can flow out of $\Omega$. This condition is called the homogeneous Von Neumann condition and can be written as follows:

$$
\left.(\nabla V)\right|_{\partial \Omega} \cdot \mathbf{e}_{\Omega}=0
$$

where $\mathbf{e}_{\Omega}$ is the outward surface normal perpendicular to $\partial \Omega$ as illustrated by figure 1 .

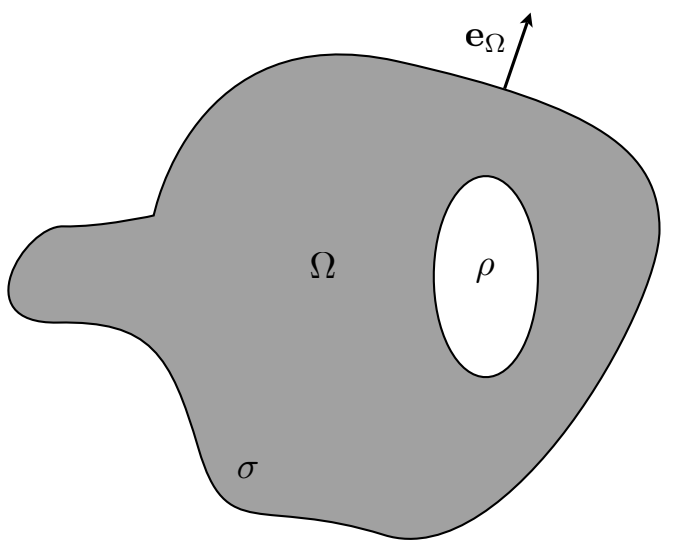

Figure 1. A homogenous 2D conductor $\Omega$ with boundary $\partial \Omega$, source distribution $\rho$, constant conductivity $\sigma$ and outward surface normal $\mathbf{e}_{\Omega}$.

The problem $\mathcal{P}$ that we shall consider is the following:

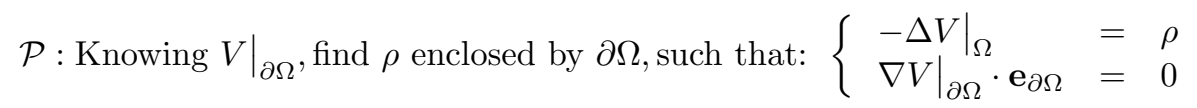

\section{GENERALIZED MEASURES}

We would like to obtain a measure which yields information about the source distribution without having to know the manifestation of $\rho$ in $\Omega$. For this purpose we use analytic functions, that is, functions whose Laplacian is zero in $\Omega$. We observe that if $V$ is known on the boundary $\partial \Omega$, then we can exactly calculate $\langle\psi, \rho\rangle$ for well-chosen test-functions $\psi$.

This can be shown using Green's theorem, which states:

$$
\int_{\partial \Omega}(V \nabla \psi-\psi \nabla V) \cdot \mathbf{e}_{\Omega} d s=\int_{\Omega}(V \Delta \psi-\psi \Delta V) d \Omega .
$$


If we choose $\psi$ such that $\Delta \psi=0$ in $\Omega$ then we can deduce the following:

$$
\int_{\partial \Omega}(V \nabla \psi-\psi \nabla V) \cdot \mathbf{e}_{\Omega} d s=-\langle\psi, \rho\rangle .
$$

Moreover, when considering the boundary condition (3) we obtain:

$$
\int_{\partial \Omega}(V \nabla \psi-\psi \nabla V) \cdot \mathbf{e}_{\Omega} d s=\int_{\partial \Omega} V \nabla \psi \cdot \mathbf{e}_{\Omega} d s,
$$

Hence, we can compute the following measures:

$$
\langle\psi, \rho\rangle=-\int_{\partial \Omega} V \nabla \psi \cdot \mathbf{e}_{\Omega} d s
$$

We call the test-function $\psi$ an "analytic sensor". In this work, we opt for analytic sensors that are rational functions of $z$ and do not have any poles in $\Omega$. As we shall see, this choise is very useful for the subsequent generalized annihilation procedure. Using the analytic formalism we can, by consequence, write the following:

$$
\langle\psi, \rho\rangle=-\int_{\partial \Omega} V(x, y) \psi^{\prime}(z) d z .
$$

Unfortunately, reconstructing $\rho$ from its generalized samples is not a well-posed problem. Hence, we need to further parametrize $\rho$.

\section{SPARSE RECONSTRUCTION}

As said before, we need to further restrain $\rho$ so that $\mathcal{P}$ becomes well-posed. Here, we assume that $\rho$ is a weighted sum of point sources. Hence we can parametrize $\rho$ as follows:

$$
\rho(\mathbf{x})=\sum_{m=1}^{M} c_{m} \delta\left(\mathbf{x}-\mathbf{x}_{m}\right),
$$

with $c_{m} \in \mathbb{R}, M \in \mathbb{N}$ and $\mathbf{x}_{m} \in \mathbb{R}^{2}$. This parametrization ensures that $\mathcal{P}$ is well-posed in the sense that there is at most one $\rho$ that generates $\left.V\right|_{\partial \Omega},{ }^{8}$ if we we have at least $2 * M$ generalized measures. Thus, the goal of the inverse problem at hand is to retrieve the positions and the intensities of the point sources.

\subsection{Choice of the analytic sensors}

Now that the problem is well-formulated and well-posed, we need to choose the sensors such that the corresponding generalized samples allow us to determine algorithmically the locations and the intensities of the point sources. Moreover we would like that the reconstruction is a one-step process which is well localized since most applications assume superficial sources. Furthermore, we would like the algorithm to be numerically stable. For these purposes we choose the following family of rational analytic sensors:

$$
\psi_{a_{n}}(z)=\frac{1}{z-a_{n}}, \quad a_{n} \notin \Omega .
$$

More specifically, we will restrict further our choice of $a_{n}$ to the form $a_{n}=\alpha_{n} e^{i n \theta}$, where $n=0, \ldots, N-1$ for some $N>2 M$ and $\theta \in] 0,2 \pi\left[\right.$. The radius $\alpha_{n}$ is chosen such that $a_{n} \notin \Omega$. This set-up is depicted in the figure 2 . 


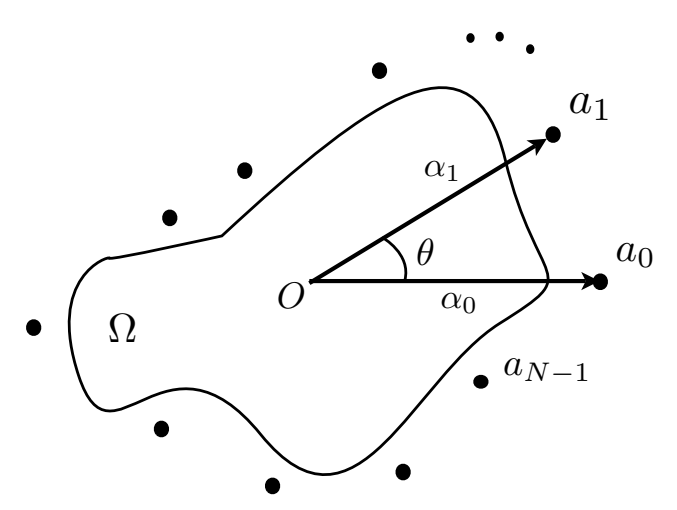

Figure 2. The poles, $a_{n}$, and their placement, outside of $\Omega$. For each pole there is a corresponding analytic sensor $\psi_{n}(z)=\frac{1}{z-a_{n}}$.

\subsection{A non-iterative solution}

Let us introduce a polynomial, $R$, whose roots are the locations of the point sources:

$$
R(X)=\prod_{m=1}^{M}\left(X-z_{m}\right)
$$

Thus, the following relationship exists between $R$ and the generalized samples $\left\langle\psi_{a_{n}}, \rho\right\rangle$ :

$$
\left\langle\psi_{a_{n}}, \rho\right\rangle=\sum_{m=1}^{M} c_{m} \psi_{a_{n}}\left(z_{m}\right)=\frac{\sum_{m=0}^{M-1} c_{m}^{\prime} e^{i m n \theta}}{R\left(a_{n}\right)} .
$$

Now, consider the coefficients $h_{k}$, such that:

$$
H(z)=\sum_{k \in \mathbf{Z}} h_{k} z^{-k}=\prod_{k=0}^{M-1}\left(1-e^{i k \theta} z^{-1}\right),
$$

then:

$$
h_{n} *\left(R\left(a_{n}\right)\left\langle\psi_{a_{n}}, \rho\right\rangle\right)=0,
$$

holds. Hence, let $R(X)=\sum_{k=0}^{M} r_{k} X^{k}$, then the coefficients $r_{k}$ are given by the linear system of equations:

$$
\sum_{k^{\prime}=0}^{M} A_{n, k^{\prime}} r_{k^{\prime}}=0
$$

with $A_{n, k^{\prime}}=h_{n} *\left\langle\psi_{a_{n}}, \rho\right\rangle a_{n}^{k^{\prime}}, n \in \mathbf{Z}$. Given that there are no more than $M$ unknown poles, it suffices to have (16) for $M$ different values of $n$ to find $r_{k}$ (up to a factor). Since $h$ is often of length $M$, the knowledge of $2 M$ consecutive values of $\langle\psi, \rho\rangle$ is sufficient to find $r_{k}$, and thus $z_{k}$ by rooting the polynomial $\mathrm{R}(\mathrm{X})$, uniquely. Hence, this yields a non-iterative algorithm to localize the point sources based on the measures computed in (8). 
Now that we know the locations of the point sources, we need to determine their strengths, $c_{m}$. From the generalized samples, we see that:

$$
\begin{aligned}
\left\langle\psi_{a_{n}}, \rho\right\rangle & =\left\langle\frac{1}{z-a_{n}}, \sum_{m=1}^{M} c_{m} \delta\left(\mathbf{x}-\mathbf{x}_{\mathbf{m}}\right)\right\rangle \\
& =\sum_{m=1}^{M} \frac{c_{m}}{z_{m}-a_{n}} .
\end{aligned}
$$

Using equation 8, we can compute $\left\langle\psi_{a_{n}}, \rho\right\rangle$. Thus, the generalized samples depend linearly on the strengths $c_{m}$. Hence, determining the strengths boils down to solving the following system of equations:

$$
\sum_{m=1}^{M} \frac{c_{m}}{z_{m}-a_{n}}=\left\langle\psi_{a_{n}}, \rho\right\rangle, \quad n \in\{0, \cdots, N-1\} .
$$

Figure 3 schematizes the complete algorithm, that is the localization of the point sources and the determination of the corresponding strengths.

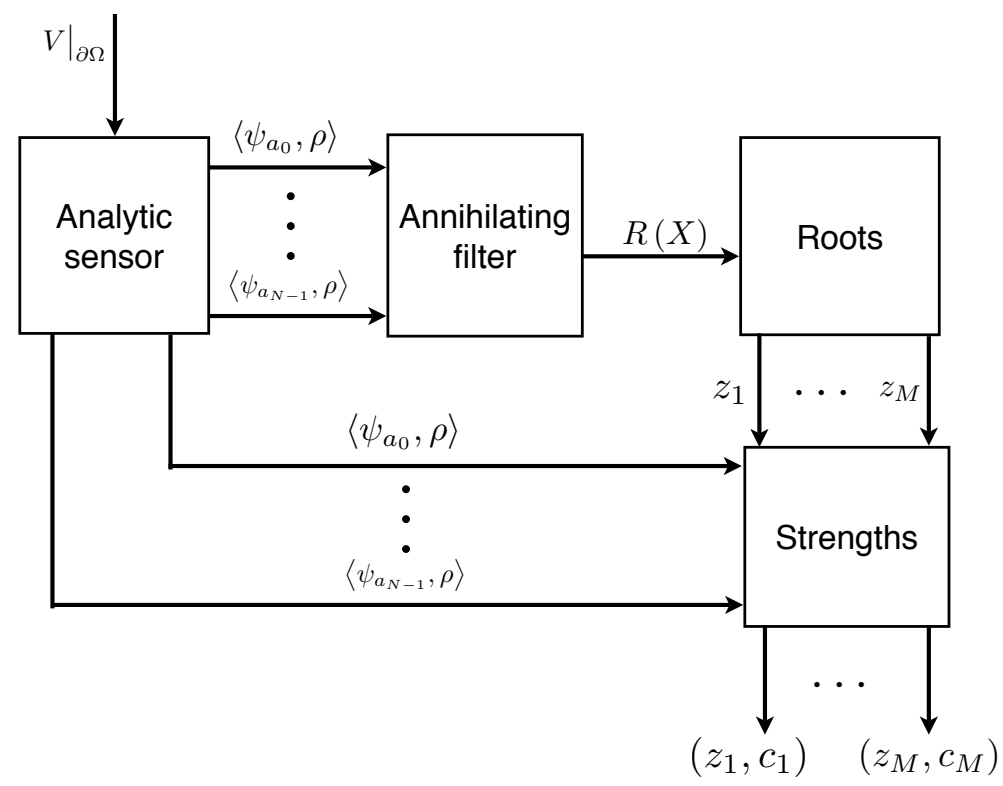

Figure 3. A flow-chart of the proposed algorithm to determine the locations of the point sources and their corresponding strengths.

\section{SIMULATIONS}

We consider a circular domain $\Omega$ and 3 point sources located at $\left(x_{1}, y_{1}\right)=(-0.8,0),\left(x_{2}, y_{2}\right)=(0.7,0.3)$ and $\left(x_{1}, y_{1}\right)=(0,-0.7)$. Next, we add Gaussian noise, with expected value 0 , to the generalized measures such that the signal-to-noise ratio(SNR) is $20 \mathrm{~dB}$. Moreover, we used the following 32 analytic sensors:

$$
\psi_{n}(z)=\frac{1}{z-1.1 e^{i \frac{\pi}{16} n}}, \quad n=0, \ldots, 31 .
$$

The estimated locations are shown in figure 4.

We see that the algorithm is able the distinguish the "active regions". In any case, in the absence of noise, the locations are exactly reconstructed. We also note that the positions of the singularities are important. That is, the closer a point source is located to a singularity the better the associated analytic sensor will be able to distinguish the point source. Hence the proposed algorithm has a local behavior which allows for local sensing. 


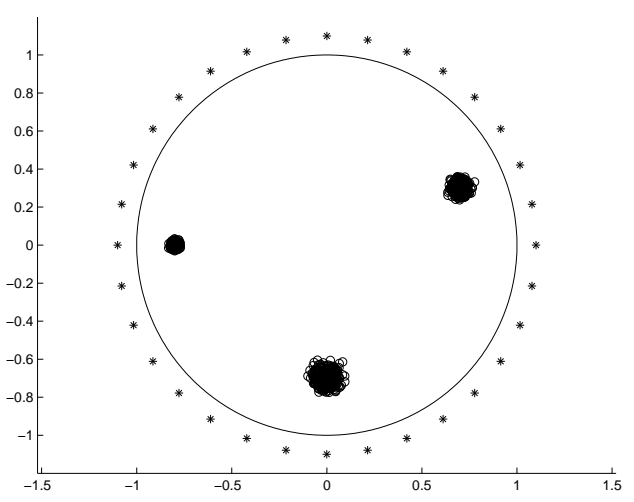

Figure 4. A scatterplot of the estimated locations of 3 point sources located at $\left(x_{1}, y_{1}\right)=(-0.8,0),\left(x_{2}, y_{2}\right)=(0.7,0.3)$ and $\left(x_{1}, y_{1}\right)=(0,-0.7)$. The noise added to the generalized measures is additive Gaussian noise such that the estimation is performed at $20 \mathrm{~dB}$.

\section{Acknowledgements}

The authors would like to thank the following institutions for their financial support: Center for Biomedical Imaging (CIBM) of the Geneva-Lausanne Universities, EPFL, foundations Leenaards and Louis-Jeantet and the Swiss National Science Foundation under grant 200020-109415. We also thank Prof. M. Unser for his support and useful discussions.

\section{REFERENCES}

1. M. Vetterli, P. Marzilliano, and T. Blu, "Sampling signals with finite rate of innovation," IEEE Transactions on Signal Processing 50(6), 2002.

2. A. N. Tikhonov, "On the stability of inverse problems," Dokl. Akad. Nauk SSSR 39(5), pp. 195-198, 1943.

3. C. M. Michel, M. M. Murray, G. Lantz, S. Gonzalez, L. Spinelli, and R. Grave de Peralta, "EEG source imaging," Clin Neurophysiol 115, pp. 2195-2222, 2004.

4. K. Miller, "Stabilized numerical prolongation with poles," SIAM J. Appl. Math. 18, pp. 346-363, 1970.

5. A. El Badia and T. Ha-Duong, "An inverse source problem in potential analysis," Inverse Problems 16, pp. 651-663, 2000.

6. J. R. Cannon and R. E. Ewing, "The locations and strengths of points sources," Improperly Posed Boundary Value Problems (Research Notes in Mathematics) 1, pp. 39-53, 1975.

7. S. Vessela, "Locations and strengths of point sources: stability estimates," Inverse Problems 8, pp. 911-917, 1992.

8. L. Baratchart, A. Ben Abda, F. Ben Hassen, and J. Leblond, "Recovery of pointwise sources or small inclusions in 2D domains and rational approximation," Inverse problems 21, pp. 51-74, 2005. 